\title{
ON A CLASS OF PATHOLOGICAL FUNCTIONS
}

\section{SEYMOUR GINSBURG}

We study the problem of approximating a function from a separable metric space $D$ to a separable metric space $Z$ by functions $g$ from $D$ to $Z$ which have the property there is no set $E$ of power $2^{N_{0}}$ such that $g$ is a homeomorphism of $E$ onto $g(E)$. Theorem 1 asserts that any one to one function may be approximated by such a function. Numerous continuous functions may be approximated by such functions which, moreover, are periodic of preassigned period $n$ (Theorem $4)$.

All functions mentioned are from a separable metric space into a separable metric space.

Definition. A one to one function $f$ of $D$ into $Z$ is a dishomeomorphism if $D$ contains no subset $E$, of power $2^{N_{0}}$, such that $f$ is a homeomorphism of $E$ onto $f(E)$.

The proof of the existence of a dishomeomorphism was given for the first time in [2].

By $R$ is meant the set of real numbers, ordered in the natural manner. By a linear set is meant a subset of $R$.

Definition. A one to one function $f$ of a linear set $E$ into $R$ is a dissimilarity transformation (on $E$ ) if $E$ contains no subset $D$, of power $2^{\aleph_{0}}$, such that $f$ is a similarity transformation of $D$ onto $f(D)$.

From the definitions there readily follows

Lemma 1. If $E$ is of power $<2 \mathbb{N}_{0}$, then each one to one function $f$ of $E$ into $Z$ is a dishomeomorphism. If $E$ and $f(E)$ are linear sets, then $f$ is also a dissimilarity transformation.

It is easily seen that a necessary and sufficient condition that each one to one function $f$, of $E$ into $E$, for which the power of the set $\{x \mid f(x)=x, x \in E\}$ is $\left\langle 2^{N_{0}}\right.$, be a dissimilarity transformation, is that $E$ have property $A .^{1}$

The following known result will be used [1]:

LAVRentiefF's Theorem. Let $D$ be a subset of $E$. If $f$ is a homeomorphism of $D$ into a complete space $C$, then $f$ can be extended to be a homeomorphism of $a G_{\delta}$ set $B$, containing $D$, into $C$.

Let $f$ be a function from $E$ into $D$. We shall say that a function $g$

Received by the editors December 8, 1954.

${ }^{1}$ A set $E$ has property $A$ if $E$ is a linear set of power 2No with the property that no two disjoint subsets of $E$, of power $2{ }^{N 0}$ each, are similar. 
of $E$ into $D$-approximates $f$ if $d(f(x), g(x))<\epsilon$ for each $x$ in $E, d$ being the metric on $D$.

Lemma 2. Let $f$ be a mapping of $D$ into a complete space $Z$ such that for each element $y$ in $E=f(D), f^{-1}(y)$ is a denumerable set. ${ }^{2}$ If each element of $E$ is a c-condensation point of $E,{ }^{3}$ then for each $\epsilon>0$ there exists a dishomeomorphism $g$ of $D$ onto $E$ which $\epsilon$-approximates $f$.

Proof. For each element $y$ in $E$ let $S(y)$ denote the sphere in $E$, of radius $\epsilon / 2$ and center $y$. Since each element of $S(y)$ is a $c$-condensation point of $E$, the power of $S(y)$ is $2^{N_{0}}$. Furthermore, since $f^{-1}(y)$ is denumerable, $f^{-1}[S(y)]$ is of power $2^{\aleph_{0}}$.

Denote by $F$ the set of all those couples $(f, B)$, where $B$ is a $G_{\delta}$ of $D$ of power $2^{\aleph_{0}}$, and $f$ is a homeomorphism of $B$ into $Z$. Since $D$ and $Z$ are separable and of power $2^{\boldsymbol{N}_{0}}$ each, the power of the set $F$ is $2^{\mathrm{N}_{0}}$. Well order the elements of $D, E$, and $F$ into the sequences $\left\{u_{\xi}\right\}$, $\xi<\theta,\left\{v_{\xi}\right\}, \xi<\theta$, and $\left\{\left(f_{\xi}, B_{\xi}\right)\right\}, \xi<\theta$ respectively. ${ }^{4}$ Denote by $w_{0}$ the element $u_{0}$. Let $x_{0}$ be the first element in the set $S\left(f\left(w_{0}\right)\right)-\left\{f_{0}\left(w_{0}\right)\right\}$. Define $g\left(u_{0}\right)$ to be $x_{0}$. Let $z_{0}$ be the first element in the set $E-\left\{x_{0}\right\}$ and $y_{0}$ the first element in $f^{-1}\left(S\left(z_{0}\right)\right)-\left\{w_{0}, f_{0}^{-1}\left(z_{0}\right)\right\}$. Define $g\left(y_{0}\right)$ to be $z_{0}$. Note that $d\left(f\left(w_{0}\right), g\left(w_{0}\right)\right)<\epsilon$ and $d\left(f\left(y_{0}\right), g\left(y_{0}\right)\right)<\epsilon$. Continuing by induction suppose that the elements $w_{\xi}, x_{\xi}=g\left(w_{\xi}\right), y_{\xi}$, and $z_{\xi}=g\left(y_{\xi}\right)$ have been defined for each $\xi<\alpha$. Let $w_{\alpha}$ be the first element in the set

$$
D-\left\{w_{\xi}, y_{\xi} \mid \xi<\alpha\right\}
$$

and $x_{\alpha}$ the first element in the set

$$
S\left(f\left(w_{\alpha}\right)\right)-\left[\left\{x_{\xi}, z_{\xi} \mid \xi<\alpha\right\} \cup\left\{f_{\xi}\left(w_{\alpha}\right) \mid \xi \leqq \alpha\right\}\right] .
$$

Define $g\left(w_{\alpha}\right)$ to be $x_{\alpha}$. Let $z_{\alpha}$ be the first element in the set

$$
E-\left[\left\{x_{\xi}, z_{\xi} \mid \xi<\alpha\right\} \cup\left\{x_{\alpha}\right\}\right] .
$$

Let $y_{\alpha}$ be the first element in

$$
f^{-1}\left(S\left(z_{\alpha}\right)\right)-\left[\left\{w_{\xi}, y_{\xi} \mid \xi<\alpha\right\} \cup\left\{f_{\xi}^{-1}\left(z_{\alpha}\right) \mid \xi \leqq \alpha\right\} \cup\left\{w_{\alpha}\right\}\right] .
$$

Since the set in the brackets is of power $\left\langle 2^{\mathrm{N}_{0}}, y_{\alpha}\right.$ exists. Define $g\left(y_{\alpha}\right)$ to be $z_{\alpha}$. Note that $d\left(f\left(w_{\alpha}\right), g\left(w_{\alpha}\right)\right)<\epsilon$ and $d\left(f\left(y_{\alpha}\right), g\left(y_{\alpha}\right)\right)<\epsilon$.

Clearly the function $g$ is one to one. From (2) and (4) it follows

${ }^{2}$ If $f$ maps $A$ into $B$, then by $f^{-1}(C), C$ being a subset of $B$, is meant $\{x \mid x \in A, f(x) \epsilon C\}$.

${ }^{3} p$ is a $c$-condensation point of $E$ if each open set containing $p$ meets $E$ in 2 so $_{0}$ elements.

${ }^{4} \mathrm{By} \theta$ is meant the smallest ordinal number whose power is $2 \mathrm{No}$. 
that for $\alpha \geqq \xi, g\left(w_{\alpha}\right) \neq f_{\xi}\left(w_{\alpha}\right)$ and $g\left(y_{\alpha}\right) \neq f_{\xi}\left(y_{\alpha}\right)$. Hence

$$
\text { the set }\left\{x \mid f_{\xi}(x)=g(x), x \in B_{\xi}\right\} \text { is of power }<2 \text { so. }
$$

From (1) for each element $u_{\nu}$ of $D, u_{\nu}$ is in the set $\left\{w_{\xi}, y_{\xi} \mid \xi \leqq \nu\right\}$. From (3) for each element $v_{\nu}$ of $E$, $v_{\nu}$ is in the set $\left\{x_{\xi}, z_{\xi} \mid \xi \leqq \nu\right\}$. Consequently $g$ is a one to one function of $D$ onto $E$ which $\epsilon$-approximates $f$.

We now show that $g$ is a dishomeomorphism. For suppose the contrary. Then there exists a subset $H$, of power $2 \mathrm{~s}_{0}$, of $D$ such that $g$ is a dishomeomorphism of $H$ onto $g(H)$. Let $h$ be the function which is defined by $h(x)=g(x)$ for $x$ in $H$. By Lavrentieff's Theorem, $h$ may be extended to be a homeomorphism $k$ of a $G_{\delta}$ set $M$, containing $H$, into $E$. This however contradicts (5) since $k$ and $g$ coincide on a set of power $2^{N_{0}}$. Therefore $g$ must be a dishomeomorphism. Q.E.D.

Suppose that $f$ is a one to one mapping of $Y$ into $Z$. Let $E$ be the set of $c$-condensation points of $f(Y)$ which are in $f(Y)$ and let $G=f(Y)$ $-E$. As is well known the power of $G$ is $\left\langle 2^{N_{0}}\right.$, and each element of $E$ is a $c$-condensation point of $E$. Let $D=f^{-1}(E)$. By Lemma 2, for $\epsilon>0$ there exists a dishomeomorphism $g$ of $D$ onto $E$ which $\epsilon$-approximates $f$. For each element $x$ in $f^{-1}(G)=Y-D$, let $g(x)=f(x)$. Now the following is easily seen.

Lemma 3. Let $D$ be the union of two disjoint sets $F$ and $G$. $A$ necessary and sufficient condition that a one to one function $f$ of $D$ into $E$ be a dishomeomorphism is that $f$ be a dishomeomorphism of each of the sets $F$ and $G$.

In view of Lemma 3 and the preceding discussion we obtain

Theorem 1. For each one to one mapping $f$ of $D$ into $E$, and for each $\epsilon>0$, there exists a dishomeomorphism $g$ of $D$ onto $f(D)$ which $\epsilon-a p-$ proximates $f$.

COROllary 1. Each one to one function $f$ of $D$ into $E$ is the limit of a uniformly convergent sequence of dishomeomorphisms of $D$ onto $f(D)$.

Corollary 2. Let $D$ be a subset of $E$ and $g$ a dishomeomorphism of $D$ onto the subset $F$ of $G$. Then $g$ can be extended to be a dishomeomorphism of $E$ onto $G$ if and only if the two sets $E-D$ and $G-g(D)$ are of the same power.

Suppose that $f$ is a similarity transformation of a linear set $E$, of power $2^{\aleph_{0}}$, into $R$. The set $D$ of points of discontinuity of $f$ are enumerable. Thus $f$ is a continuous similarity transformation of the set $E-D$, of power $2^{\mathrm{N}_{0}}$, onto $f(E-D)=C$. The function $f^{-1}$ is a similarity trans- 
formation defined on $C$. Let $B$ be the denumerable set of points of discontinuity of $f^{-1}$ on $C$. Then $f^{-1}$ is a continuous similarity transformation of the set $C-B$, of power $2^{\aleph_{0}}$, onto $f^{-1}(C-B)=A$. Consequently $f$ is both a similarity transformation and a homeomorphism of $A$ onto $f(A)$. From this it follows that if $f$ is a dishomeomorphism of a linear set, then $f$ is a dissimilarity transformation. Hence

Corollary 3. For each one to one mapping of a linear set $E$ into $R$, and for each $\epsilon>0$, there exists a function $\mathrm{g}$, which is both a dishomeomorphism and a dissimilarity transformation, of $E$ onto $f(E)$. Furthermore, $g$ e-approximates $f$.

If, in Lemma 3 and Corollary 2, all sets are linear, then "dishomeomorphism" may be replaced by "dissimilarity transformation."

By a procedure quite analogous to Theorem 1 the following result may be proved.

THeOREM 2. Let $f$ be a mapping of $D$ into itself such that for each element $y$ in $E=f(D), f^{-1}(y)$ is a denumerable set. If each element of $E$ is a c-condensation point of $E$, then for each $\epsilon>0$ there exists a dishomeomorphism $g$ of $D$ onto $E$ which $\epsilon$-approximates $f$ and also has the property that there is no element $x$ in $D$ and no positive integer $n$ such that $f^{n}(x)=x$.

COROLLARy. Let $f$ be a mapping of $D$ into itself such that for each element $y$ in $E=f(D), f^{-1}(y)$ is a denumerable set. Suppose that there is no element $x$ in $D$ and positive integer $n$ such that $f^{n}(x)=x$. Then there exists a dishomeomorphism $g$ of $D$ onto $E$ which $\epsilon$-approximates $f$ and also has the property that there is no element $x$ in $D$ and no positive integer $n$ such that $f^{n}(x)=x$.

A function $f$ of $A$ into $A$ which is not the identity is said to be of period two if $f^{2}(x)=x$ for every element $x$ in $A .^{5} f$ is said to be strongly of period $n>0$ if $f^{n}(x)=x$ for each $x$ in $D$, and for no $0<j<n$ does there exist an $x$ so that $f^{j}(x)=x$.

THEOREM 3. Let $f$ be a one to one function of a nondenumerable space $A$ into itself with the following property: For no odd integer $>5$ is there an element $x$ in $A$ such that $f^{n}(x)=x$ and $f^{j}(x) \neq x$ for each $j=1,2, \cdots$, $n-1$. Let $\epsilon$ be any positive number which has the property that $d\left(x, f^{2}(x)\right)$ $<\epsilon / 2$ for each $x$ in $A$. Then there exists a dishomeomorphism $g$ of $A$ onto $A$ which is of period two and $\epsilon$-approximates $f$.

Proof. Since $A$ is a separable metric space there exists a denumer-

${ }^{5}$ For each function $f, f^{0}(x)=x$ and $f^{n+1}(x)=f\left[f^{n}(x)\right]$. Let $f^{-n}(x)=\left(f^{n}\right)^{-1}(x)$. 
able set of spheres $\left\{S_{n} \mid n<\omega\right\}$, of diameter $<\epsilon / 2$, which form a basis for the topology of $A$. Denote by $K_{n}^{m}$ the set

$$
K_{n}^{m}=\left\{x \mid x \in S_{m}, f(x) \in S_{n}\right\} .
$$

Evidently $A=\bigcup_{m, n} K_{n}^{m}$. Relabel the $K_{n}^{m}$ as $B_{i}, i<\omega$, i.e., $\left\{K_{n}^{m} \mid m\right.$, $n<\omega\}=\left\{B_{i} \mid i<\omega\right\}$. Let $C_{i}=f\left(B_{i}\right)$. Let $P_{i}$ and $Q_{i}$ be the set of $c$-condensation points of $B_{i}$ and $C_{i}$ which are in $B_{i}$ and $C_{i}$ respectively. Let

$$
U=\bigcup_{i}\left[\left(B_{i}-P_{i}\right) \cup\left(C_{i}-Q_{i}\right)\right]
$$

and

$$
V=\left\{f^{r}(x) \mid x \in U, r<\omega\right\} \cup\left\{f^{-r}(x) \mid x \in U, r<\omega\right\} .
$$

As is easily seen, the power of $V$ is $\left\langle 2 \boldsymbol{N}^{\text {No }}\right.$. Let $D_{i}=B_{i}-V$ and $E_{i}=C_{i}$ $-V$. Thus $f\left(D_{i}\right)=E_{i}$. Notice that if $x$ is in $D_{i}$, then each element of $D_{i}$ is a $c$-condensation point of $D_{i}$ (thus, if $D_{i}$ is nonempty, then $D_{i}$ is of power $\left.2^{N_{0}}\right)$, and each element of $E_{i}$ is a $c$-condensation point of $E_{i}$.

We now define $g$ on $V$. Let $y$ be any element of $V$ and consider the sequence

$$
\cdots f^{-n}(y), \cdots, f^{-1}(y), y, f(y), \cdots, f^{m}(y), \cdots .
$$

If for some $n$ the element $f^{-1}\left[f^{-n}(y)\right]$ does not exist, then without loss of generality we may assume that $n=0$, i.e., $f^{-1}(y)$ does not exist. In this case (1) becomes

$$
y, f(y), \cdots, f^{m}(y), \cdots .
$$

By assumption there is no odd integer $n>5$ and no element $x$ in $A$ such that $f^{n}(x)=x$ and $f^{j}(x) \neq x$ for each $j=1,2, \cdots, n-1$. If the elements in the sequence (1) or (2) are not all distinct, then there are $1,3,5$, or an even number of distinct elements in (1) or (2). Let $J_{y}$ be the set of elements in (1) or (2). For any two elements $y$ and $z$ in $V$ either $J_{y}=J_{z}$ or else $J_{y}$ and $J_{z}$ are distinct. If the power of $J_{y}$ is either infinite or even, let $g\left[f^{2 n}(y)\right]=f^{2 n+1}(y)$ and $g\left[f^{2 n+1}(y)\right]$ $=f^{2 n}(y)$ for $n \geqq 0$, and let $g\left[f^{-2 n}(y)\right]=f^{1-2 n}(y)$ and $g\left[f^{1-2 n}(y)\right]=f^{-2 n}(y)$ for $n>0$. Suppose that $J_{y}$ contains just three elements, $y, f(y)$, and $f^{2}(y)$, i.e., $f^{3}(y)=y$. Let $g(y)=y, g[f(y)]=f^{2}(y)$, and $g\left[f^{2}(y)\right]=f(y)$. On calculating $d(f(x), g(x))$ for $x$ in $J_{y}$ we have

$$
d(f[f(y)], g[f(y)])=0 \text { and } d\left(f\left[f^{2}(y)\right], g\left[f^{2}(y)\right]\right)=d\left(f^{3}(y), f(y)\right)<\epsilon / 2 \text {. }
$$

Now $d\left(y, f^{2}(y)\right)<\epsilon / 2$ and $d\left(f^{2}(y), f(y)\right)=d\left(f^{2}(y), f^{4}(y)\right)<\epsilon / 2$. Thus $d[f(y), g(y)]=d(f(y), y) \leqq d\left(y, f^{2}(y)\right)+d\left(f^{2}(y), f(y)\right)<\epsilon / 2+\epsilon / 2=\epsilon$. 
Suppose that $J_{y}$ contains just five elements, $y, f(y), f^{2}(y), f^{3}(y)$, and $f^{4}(y)$. Since $d\left(f(y), f^{3}(y)\right)<\epsilon / 2$ and $d\left(f^{3}(y), y\right)<\epsilon / 2, d(y, f(y))<\epsilon$. Likewise $d(z, f(z))<\epsilon$ for each $z$ in $J_{y}$. Define $g(y)=f(y), g[f(y)]$ $=f^{3}(y), g\left[f^{3}(y)\right]=f(y), g\left[f^{2}(y)\right]=f^{4}(y)$, and $g\left[f^{4}(y)\right]=f^{2}(y)$. For each of the five elements, $d(f(x), g(x))<\epsilon$. Suppose that $J_{y}$ contains but one element. If $V \neq A$ let $g(y)=y$. Suppose that $V=A$, i.e., $A$ is of power $<2^{\aleph_{0}}$. If, for some $x, J_{x}$ contains more than one element, let $g(y)=y$. Suppose that $J_{x}=\{x\}$ for each $x$ in $V$. Since $V$ is nondenumerable, there exist two elements $u$ and $v$ in $V$ such that $d(u, v)<\epsilon$. Let $g(u)=v, g(v)=u$, and $g(x)=x$ for each $x$ in $V-\{u, v\}$. If $V=A$, then $g$ is a well defined function of $V$ onto $V$, of period two, which $\epsilon$-approximates $f$. By Lemma $1, g$ is a dishomeomorphism. If $V \neq A$, then $g$ is a function of period $\leqq 2$.

Suppose that $A$ is of power $2^{N_{0}}$. Let $W=A-V$. We modify the demonstration given in Theorem 1 . Denote by $F$ the set of those couples $(f, B)$, where $B$ is a $G_{\delta}$ of $A$ of power $2^{\mathrm{N}_{0}}$, and $f$ is a homeomorphism of $B$ into $A^{*}, A^{*}$ being the completion of $A$. Well order the elements of $W$ and of $F$ into the sequence $\left\{u_{\xi}\right\}, \xi<\theta$, and $\left\{\left(f_{\xi}, B_{\xi}\right)\right\}$, $\xi<\theta$, respectively. Suppose that the elements

$$
w_{\xi}, x_{\xi}=g\left(w_{\xi}\right) \text {, and } z_{\xi}=g\left(y_{\xi}\right)
$$

have been defined for $\xi<\alpha$. Let $w_{\alpha}$ be the first element in

$$
W-\left\{w_{\xi}, x_{\xi}, y_{\xi}, z_{\xi} \mid \xi<\alpha\right\} \text {. }
$$

There exists a set $D_{i}$, say $D_{r(\alpha)}$, which contains $w_{\alpha}$. Let $x_{\alpha}$ be the first element in the set

$$
\begin{aligned}
E_{r(\alpha)}-\left[\left\{w_{\xi}, x_{\xi}, y_{\xi}, z_{\xi} \mid \xi<\alpha\right\}\right. & \\
& \left.\cup\left\{w_{\alpha}\right\} \cup\left\{f_{\xi}\left(w_{\alpha}\right) \mid \xi \leqq \alpha\right\} \cup\left\{f_{\xi}^{-1}\left(w_{\alpha}\right) \mid \xi \leqq \alpha\right\}\right] .
\end{aligned}
$$

The element $x_{\alpha}$ certainly exists since $D_{r(\alpha)}$, thus $E_{r(\alpha)}$, is of power $2^{\aleph_{0}}$, whereas the set in the brackets is of power $<2 \boldsymbol{N}_{0}$. Define $g\left(w_{\alpha}\right)$ to be $x_{\alpha}$ and $g\left(x_{\alpha}\right)$ to be $w_{\alpha}$. Since $f\left(w_{\alpha}\right)$ is in $E_{r(\alpha)}$ and the diameter of $E_{r(\alpha)}$ is $<\epsilon / 2, d\left(f\left(w_{\alpha}\right), g\left(w_{\alpha}\right)\right)<\epsilon$. Now there exists an element $a_{\alpha}$ in $D_{r(\alpha)}$ such that $f\left(a_{\alpha}\right)=x_{\alpha}$. Thus

$$
\begin{gathered}
d\left(f\left(x_{\alpha}\right), g\left(x_{\alpha}\right)\right)=d\left(f^{2}\left(a_{\alpha}\right), w_{\alpha}\right) \leqq d\left(a_{\alpha}, w_{\alpha}\right)+d\left(a_{\alpha}, f^{2}\left(a_{\alpha}\right)\right) \\
<\epsilon / 2+\epsilon / 2=\epsilon .
\end{gathered}
$$

Let $z_{\alpha}$ be the first element in the set

$$
W-\left[\left\{w_{\xi}, x_{\xi}, y_{\xi}, z_{\xi} \mid \xi<\alpha\right\} \cup\left\{w_{\alpha}, x_{\alpha}\right\}\right] .
$$

$f\left(z_{\alpha}\right)$ is in one of the sets $D_{i}$, say $D_{s(\alpha)}$. Let $y_{\alpha}$ be the first element in the set 


$$
\begin{aligned}
D_{s(\alpha)}-\left[\left\{w_{\xi}, x_{\xi}, y_{\xi}, z_{\xi} \mid \xi<\alpha\right\}\right. & \\
\cup & \left.\left\{w_{\alpha}, x_{\alpha}, z_{\alpha}\right\} \cup\left\{f_{\xi}\left(z_{\alpha}\right), f_{\xi}^{-1}\left(z_{\alpha}\right) \mid \xi \leqq \alpha\right\}\right] .
\end{aligned}
$$

Define $g\left(y_{\alpha}\right)$ to be $z_{\alpha}$ and $g\left(z_{\alpha}\right)$ to be $y_{\alpha}$. Evidently $d\left(f\left(z_{\alpha}\right), g\left(z_{\alpha}\right)\right)$ $=d\left(f\left(z_{\alpha}\right), y_{\alpha}\right)<\epsilon$. Also

$$
d\left(f\left(y_{\alpha}\right), g\left(y_{\alpha}\right)\right)=d\left(f\left(y_{\alpha}\right), z_{\alpha}\right) \leqq d\left(f\left(y_{\alpha}\right), f^{2}\left(z_{\alpha}\right)\right)+d\left(z_{\alpha}, f^{2}\left(z_{\alpha}\right)\right)<\epsilon .
$$

The function $g$ is well defined, of period two (since $g$ is not the identity mapping), and $\epsilon$-approximates $f$. From (3) and (5), $g$ maps $W$ onto $W$, thus $A$ onto $A$. As in Theorem 1 we see that $g$ is a dishomeomorphism. Q.E.D.

Corollary. Each function, of period two, of a nondenumerable space $A$ into itself is the limit of a uniformly convergent sequence of functions $\left\{f_{n}(x)\right\}$, each function being of period two, and each function being a dishomeomorphism of $A$ onto $A$.

If "nondenumerable" is removed from the hypothesis of Theorem 3 , then the conclusion is no longer valid. For example, let $A$ be the set of positive integers, $f$ the identity, and $\epsilon=1 / 3$. The only function which $\epsilon$-approximates $f$ is $f$ itself. However $f$ is not of period two.

The condition " $n>5$ " cannot be removed from Theorem 3 . For example, consider a regular heptagon inscribed in the circle of radius one and center the origin, the vertices in clockwise order being $x_{1}, x_{6}, x_{4}, x_{2}, x_{7}, x_{5}$, and $x_{3}$. Let $A=\left\{x_{i} \mid i \leqq 7\right\} \cup M \cup N$, where $M=\{(4, y) \mid 0 \leqq y \leqq 1 / 2\}$ and $N=\{(41 / 10, y) \mid 0 \leqq y \leqq 1 / 2\}$. Let $\epsilon / 2=d\left(x_{1}, x_{6}\right)+1 / 1000$. By trigonometry, $d\left(x_{1}, x_{2}\right)>\epsilon$. Let $f\left(x_{7}\right)=x_{1}$ and for $i<7$ let $f\left(x_{i}\right)=x_{i+1}$. Let $f(4, y)=(41 / 10, y)$ and $f(41 / 10, y)$ $=(4, y)$. As is easily seen, any function $g$, of period two, which $\epsilon$-approximates $f$, maps a vertex onto a vertex. Hence for at least one $k, g\left(x_{k}\right)=x_{k}$. Then $d\left(f\left(x_{k}\right), g\left(x_{k}\right)\right)>\epsilon$, i.e., $g$ goes not $\epsilon$-approximate $f$. In view of the previous example, an arbitrary function of a space $D$ need not be $\epsilon$-approximated by a function which is strongly of period $n$. However, we do have

Theorem 4. Suppose that each element of $D$ is a c-condensation point of $D$. Let $n$ be any integer $>1$ and let $f$ be a continuous function of $D$ into itself such that for each $x$ in $D$, (i) $d\left(x, f^{n}(x)\right)<\epsilon / 2$, and (ii) $f^{-1}(x)$ is of power $<2^{N_{0}}$. Then there exists a function $g$, strongly of period $n$, which $\epsilon$-approximates $f$.

Proof. Let $S(x)$ denote the sphere in $D$ of radius $\epsilon$ and center $x$. Let $F$ be the set of all pairs $(h, B)$, where $B$ is a $G_{\delta}$ of $D$ and $h$ is a homeomorphism of $B$ into $E, E$ being the completion of $D$. Well order the 
elements of $D$ and $F$ into the sequences $\left\{x_{\xi}\right\}, \xi<\theta$, and $\left(f_{\xi}, B_{\xi}\right)$, $\xi<\theta$, respectively. For $\xi<\alpha$ suppose that $u_{i}^{\xi}, 1 \leqq i \leqq n$, have been defined so that $g\left(u_{j}^{\xi}\right)=u_{j+1}^{\xi}$ for $j<n$ and $g\left(u_{n}^{\xi}\right)=u_{1}^{\xi}$. Let $u_{1}^{\alpha}$ be the first element in $D-G_{\alpha}$, where $G_{\alpha}=\left\{u_{j}^{\xi} \mid \xi<\alpha, j \leqq n\right\}$. Let $O_{1}^{\alpha}$ be an open subset of $S\left(u_{1}^{\alpha}\right)$ containing $u_{1}^{\alpha}$ such that $f\left(O_{1}^{\alpha}\right) \subseteq S\left(f\left(u_{1}^{\alpha}\right)\right)$. The continuity of $f$ assures us that $O_{1}^{\alpha}$ exists. Since $O_{1}^{\alpha}$ is open, each element in $O_{1}^{\alpha}$ is a $c$-condensation point of $O_{1}^{\alpha}$. The continuity of $f$ and (ii) of the hypothesis imply that each point of $f\left(O_{1}^{\alpha}\right)$ is a $c$-condensation point of $f\left(O_{1}^{\alpha}\right)$. Suppose that $O_{i}^{\alpha}$ and $u_{i}^{\alpha}$ have been defined for $i<j<n$ so that $O_{i}^{\alpha}$ contains $u_{i}^{\alpha}, f\left(O_{i}^{\alpha}\right) \subseteq S\left(f\left(u_{i}^{\alpha}\right)\right)$, and each point of $f\left(O_{i}^{\alpha}\right)$ is a $c$-condensation point of $f\left(O_{i}^{\alpha}\right)$. Let $u_{j}^{\alpha}$ be the first element in

$$
f\left(O_{j-1}^{\alpha}\right)-\left[G_{\alpha} \cup\left\{u_{i}^{\alpha} \mid i<j\right\} \cup\left\{f_{\xi}\left(u_{j-1}^{\alpha}\right) \mid \xi \leqq \alpha\right\}\right] .
$$

The power condition on the sets guarantee the existence of $u_{j}^{\alpha}$. Let $O_{j}^{\alpha}, O_{j}^{\alpha} \subseteq f\left(O_{j-1}^{\alpha}\right)$ be an open set, relative to $f\left(O_{j-1}^{\alpha}\right)$, containing $u_{j}^{\alpha}$ so that $f\left(O_{j}^{\alpha}\right) \subseteq S\left(f\left(u_{j-1}^{\alpha}\right)\right)$. Since each element of $f\left(O_{j-1}^{\alpha}\right)$ is a $c$-condensation point of $f\left(O_{j-1}^{\alpha}\right)$ and $O_{j}^{\alpha}$ is open in $f\left(O_{j-1}^{\alpha}\right), O_{j}^{\alpha}$, thus $f\left(O_{j}^{\alpha}\right)$ have the same property. Let $u_{n}^{\alpha}$ be the first element in

$$
f\left(O_{n-1}^{\alpha}\right)-\left[G_{\alpha} \cup\left\{u_{i}^{\alpha} \mid i \leqq n-1\right\} \cup\left\{f_{\xi}\left(u_{n-1}^{\alpha}\right), f_{\xi}^{-1}\left(u_{1}^{\alpha}\right) \mid \xi \leqq \alpha\right\}\right] .
$$

Let $g\left(u_{i}^{\alpha}\right)=u_{i+1}^{\alpha}$ for $i<n$ and $g\left(u_{n}^{\alpha}\right)=u_{1}^{\alpha}$.

By the induction, $g$ is defined on all of $D$. Evidently $g$ is strongly of period $n$. Being periodic, $g$ maps $D$ onto $D$. Repeating the argument used in Lemma 2 in conjunction with $f_{\xi}\left(u_{j}^{\alpha}\right) \neq g\left(u_{j}^{\alpha}\right)$ for $\alpha \geqq \xi$ and $j \leqq n$, it follows that $g$ is a dishomeomorphism. For $i<n, g\left(u_{i}^{\alpha}\right)$ is in $S\left(f\left(u_{i}^{\alpha}\right)\right)$, i.e., $d\left(f\left(u_{i}^{\alpha}\right), g\left(u_{i}^{\alpha}\right)\right)<\epsilon$. Since $O_{i+1}^{\alpha} \subseteq f\left(O_{i}^{\alpha}\right)$ for $i<n-1$, there exists a $z_{\alpha}$ in $O_{1}^{\alpha}$ such that $f^{n-1}\left(z_{\alpha}\right)=u_{n}^{\alpha}$. Then

$$
\begin{aligned}
d\left(f\left(u_{n}^{\alpha}\right), g\left(u_{n}^{\alpha}\right)\right) & =d\left(f\left(u_{n}^{\alpha}\right), u_{1}^{\alpha}\right) \leqq d\left(u_{1}^{\alpha}, z_{\alpha}\right) \pm d\left(z_{\alpha}, f^{n}\left(z_{\alpha}\right)\right) \\
& <\epsilon / 2+\epsilon / 2=\epsilon,
\end{aligned}
$$

since $d\left(x, f^{n}(x)\right)<\epsilon / 2$ by hypothesis. Hence $g \epsilon$-approximates $f$.

THEOREM 5. For each infinite space $D$ and each integer $n>1$ there exists a dishomeomorphism which is strongly of period $n$ on $D$.

Proof. Let $B$ be the set of $c$-condensation points of $D$ which are in $D$. If $D$ is of power $<2^{N_{0}}$, let $A=D$. If $D$ is of power $2^{\mathrm{N}_{0}}$, let $A=(D-B) \cup F$, where $F$ is a denumerably infinite subset of $D$. In either case $A$ is an infinite set of power $<2^{N_{0}}$. Let $A$ be the union of $n$ disjoint sets $A_{i}$, each having the same power as $A$. This is possible since $A$ is infinite. For $i<n$ let $f_{i}$ be a one to one mapping of $A_{i}$ onto 
$A_{i+1}$, and let $f_{n}$ be a one to one mapping of $A_{n}$ onto $A_{1}$. For $x$ in $A_{i}$ let $f(x)=f_{i}(x)$. Since $A$ is of power $<2^{N_{0}}, f$ is a dishomeomorphism. Clearly $f$ is strongly of period $n$. If $A=D$, then $f$ is the desired function. Suppose that $A \neq D$. By Theorem 4, there exists a dishomeomorphism $g$, strongly of period $n$, on $D-A$, which $\epsilon$-approximates the identity function. The function $h$, which coincides with $f$ on $A$ and coincides with $g$ on $D-A$, is then the desired function.

We close with the following question: Given two separable metric spaces $D$ and $E$ of power $2^{\mathrm{N}_{0}}$ each, does there exist a one to one function $f$ of $D$ into $E$ so that for each subset $A$ of $D$, of power $2^{N_{0}}, A$ and $f(A)$ are not homeomorphic (not necessarily under $f$ )?

\section{BIBLIOGRAPHY}

1. M. Lavrentieff, Contribution a la théorie des ensembles homeomorphes, Fund. Math. vol. 6 (1924) p. 149.

2. W. Sierpiński and A. Zygmund, Sur une fonction que est discontinue sur tout ensemble de puissance du continu, Fund. Math. vol. 4 (1923) pp. 316-318.

UNIVERSITY OF MiAMI 